\title{
Propiedades del grafeno y sus aplicaciones en el campo energético
}

Properties of graphene and its applications in the energy field

Recibido: agosto 17 de 2018 | Revisado: setiembre 26 de 2018 | Aceptado: octubre 02 de 2018

Thauso Gad Pachamango
Bautista $^{\text {I }}$
Lily Fanny Zapata
Revoredo $^{\text {I }}$

1 Universidad de San Martín de Porres - Filial Norte, Chiclayo Perú tpachamangogob@usmp.pe lzapatar@usmp.pe

\section{RESUMEN}

En la última década se ha visto como los problemas económicos, sociales y ambientales debido a la generación y transporte de la energía eléctrica se han incrementado, esto ha conllevado a que investigadores de diversas áreas de la ingeniería se comprometan en la búsqueda de materiales eficientes para el desarrollo de fuentes de energía renovable. Diversas investigaciones dan luces que unos de los materiales que ya se están empezando a utilizar para el desarrollo de fuentes de energía renovable, es el grafeno. El grafeno es un material manométrico bidimensional, formada por una red hexagonal de átomos de carbono muy similar a un panal de abejas. En el 2004, gracias a la investigación de científicos de la Universidad de Manchester en Reino Unido se logró superar uno de los principales inconvenientes que presentaba este material, la inestabilidad termodinámica a escala manométrica fue superada y de esta manera se dio inicio a una nueva era de estudio e investigación en materiales manométricos bidimensionales. Este artículo evidencia las principales propiedades del grafeno y el comportamiento de este en el desarrollo de fuentes de energía renovables, en las cuales posteriormente al análisis de su desempeño y su impacto en comparación con las actuales fuentes de energía utilizadas, se demuestra que estamos frente a un material que es pilar fundamental en el desarrollo de nuevas fuentes de energía renovables más eficientes.

Palabras clave: grafeno, carbono, material bidimensional, energía renovable

\begin{abstract}
In the last decade it has been seen how the economic, social and environmental problems due to the generation and transportation of electric power have increased, this has led researchers from various areas of engineering to engage in the search for efficient materials for the development of renewable energy sources. Several investigations could yield helpful insights that one of the materials that are already beginning to be used for the development of renewable energy sources, is graphene. Graphene is a two-dimensional nanometric material, formed by a hexagonal network of carbon atoms very similar to a honeycomb. In 2004, thanks to the research of scientists from the University of Manchester in the United Kingdom, one of the main drawbacks of this material was overcome. Thermodynamic instability at a nanometric scale was overcome, and a new era of study and research in two-dimensional nanometric materials started. This article shows the main properties of graphene and its behavior in the development of renewable energy sources, in which after analyzing its performance and its impact compared to the current energy sources used, it shows that we are facing to a material that is a fundamental pillar in the development of new, more efficient renewable energy sources.
\end{abstract}

Key words: Graphenem carbon, two dimensional material, renewable energy

https://doi.org/10.24265/campus.2018.v23n26.08 


\section{Introducción}

El carbono, en la naturaleza, se puede presentar en cinco formas alotrópicas: el diamante, grafito, fulerenos, nanatubos y nanoespumas. Alotropía en química está referido a la existencia, especialmente en estado sólido, de dos o más formas estructurales moleculares o cristalinas de un elemento. De todas sus formas alotrópicas, la que en los últimos años ha cobrado un particular interés y ha sido ampliamente estudiada es el grafito debido que este material está constituido de láminas superpuestas de grafeno.

El grafeno, considerado el primer nanomaterial bidimensional descubierto, presenta propiedades fascinantes: conduce el calor 10 veces mejor que el cobre y la electricidad mejor que el silicio. Resiste el calor mejor que el diamante, es 100 veces más resistente que el acero. También es flexible, por lo que puede adoptar cualquier forma. Es el material más delgado y también el más ligero; es transparente y a la vez tan denso que es impermeable a los gases, incluso al formado por helio, el segundo átomo más pequeño (Chávez Castillo, 2012; Katsnelson, 2012; Castro Neto et al., 2009; Lee et al., 2008).

A causa de estas extraordinarias propiedades, el grafeno, actualmente, es el nanomaterial con mayor potencial para reemplazar al silicio, originando una nueva revolución tecnológica, así como lo fue el silicio y el germanio en su momento. Estas propiedades también hacen que el grafeno sea el material ideal para ser aplicado no solo en el campo de la electrónica, sino también la medicina, farmacéutica, energía, entre otras, las cuales se verán ampliamente beneficiadas de este novedoso nanomaterial bidimensional.

\section{Estructura del grafeno}

El grafeno es un material bidimensional nanométrico de átomos de carbono fuertemente cohesionados. La superficie del grafeno es plana. Puede presentar ligeras ondulaciones y un átomo de espesor. La disposición de los átomos se asemeja a la de un panal de abejas por su configuración atómica hexagonal. (Figura 1)

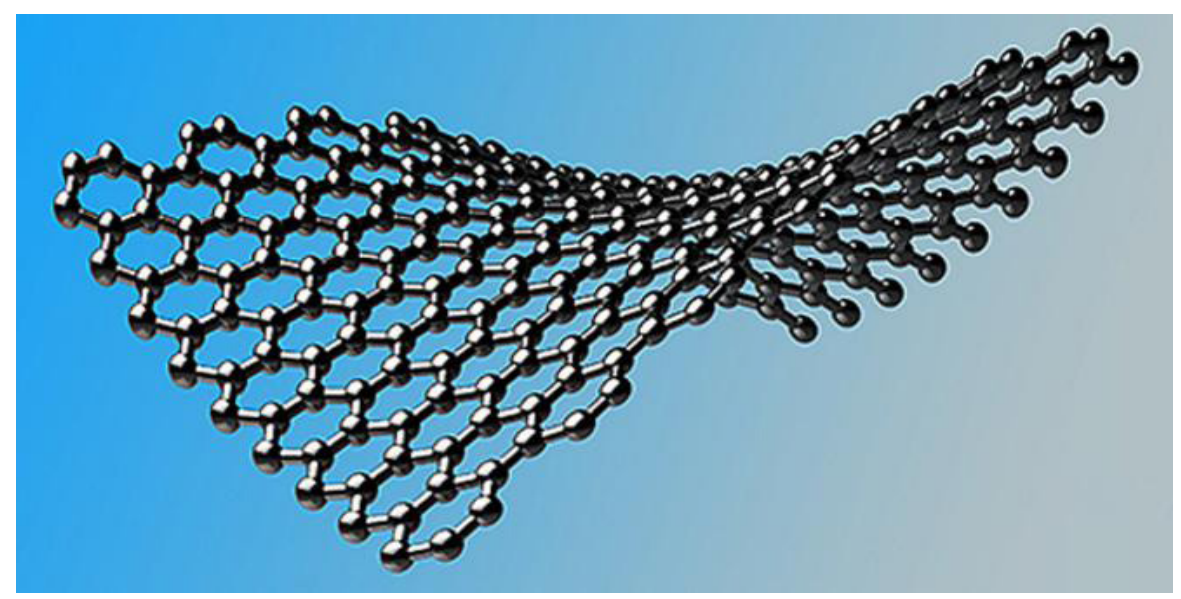

Figura 1. Estructura bidimensional del grafeno

De esta disposición atómica del carbono, se derivan propiedades electrónicas mecánicas y químicas únicas del grafeno. Extrayen- do una porción de la Figura 1, se muestra seguidamente algunas relaciones importantes observadas en los átomos de carbono. 

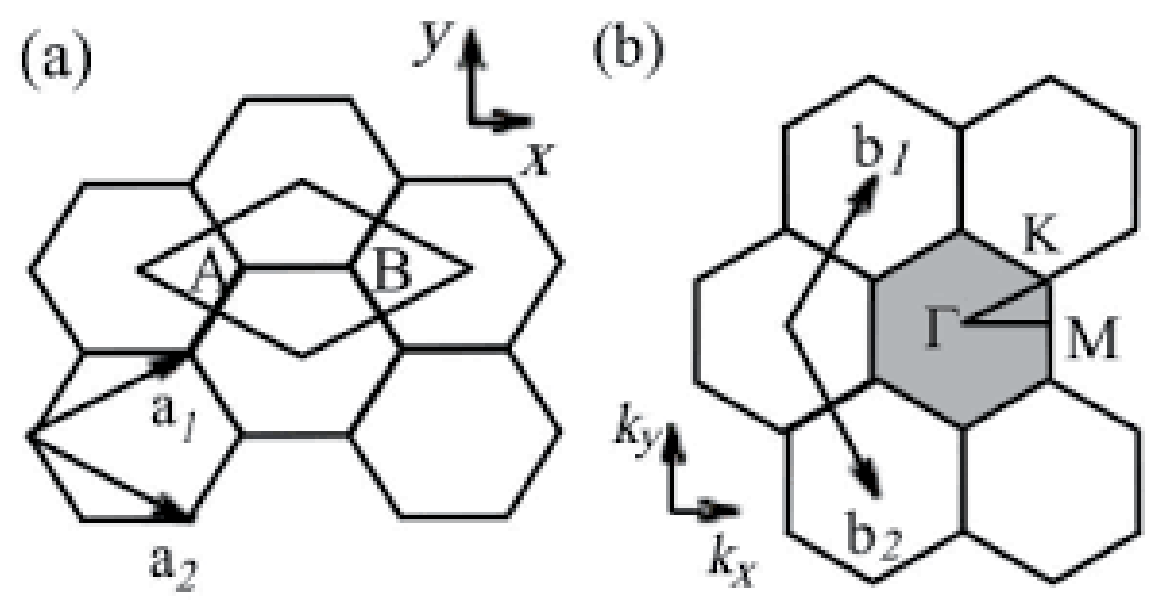

Figura 2. (a) Celda unitaria

(b) Lona de Brillouin del grateno

En la Figura 2 (a), $\vec{a}_{1}$ y $\vec{a}_{2}$ son vectores unitarios en espacio real, y en (b), $\vec{b}_{1}$ y $\vec{b}_{2}$ son vectores de la red recíproca. En las coordenadas X y Y de la figura 02, los vec- tores unitarios en espacio real $\vec{a}_{1}$ y $\vec{a}_{2}$ de la configuración hexagonal están expresadas como:

$$
\vec{a}_{1}=\left(\frac{\sqrt{3}}{2} a, \frac{a}{2}\right) \quad, \quad \vec{a}_{2}=\left(\frac{\sqrt{3}}{2} a,-\frac{a}{2}\right)
$$

Donde $a=\left|\overrightarrow{a_{1}}\right|=\left|\overrightarrow{a_{2}}\right|=1.42 \times \sqrt{3}=2.46 A$ es la constante de configuración del grafeno.

Análogamente, los vectores unitarios $\vec{b}_{1}$ y $\vec{b}_{2}$ de la red recíproca están dados por

$$
\vec{b}_{1}=\left(\frac{2 \pi}{\sqrt{3} a}, \frac{2 \pi}{a}\right) \quad, \quad \vec{b}_{2}=\left(\frac{2 \pi}{\sqrt{3} a},-\frac{2 \pi}{a}\right)
$$

Correspondiente a una constante de configuración de $\frac{4 \pi}{\sqrt{3 a}}$ en el espacio recíproco. Los tres puntos de alta simetría $\Gamma$, $\mathrm{K}$ y $\mathrm{M}$ forman un triángulo que se utiliza para calcular las relaciones de dispersión de energía, llamada también Zona de Brillouin.

Entre otros aspectos, la importancia del grafeno radica en que se puede con- siderar a este nanomaterial, como la base para la constitución de otros materiales grafiticos. Por ejemplo, si se le envuelve a manera del forro de una superficie esférica, proporciona fullerenos; si se le enrolla cilíndricamente, nanotubos; si se le superpone tridimensionalmente, grafito. En la Figura 3 se muestra las constituciones más estudiadas e investigadas actualmente. 


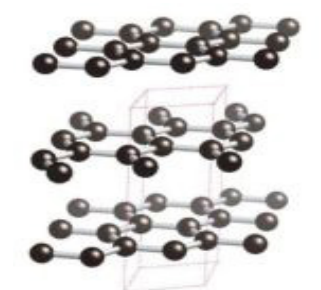

graphite

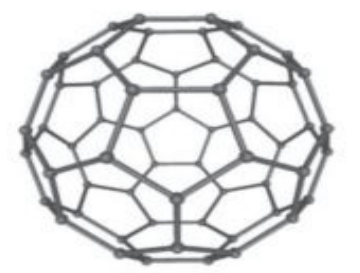

fullerene

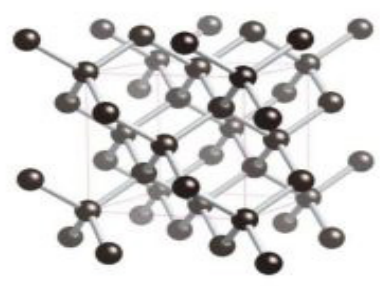

diamond

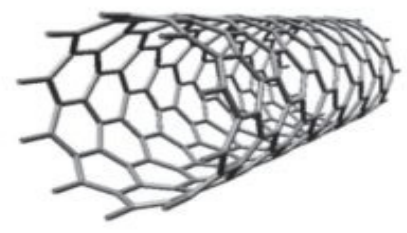

nanotube

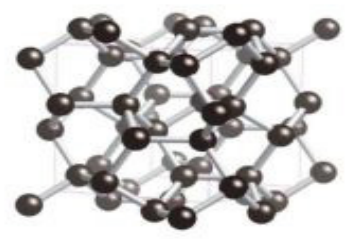

$\mathrm{BC} 8$

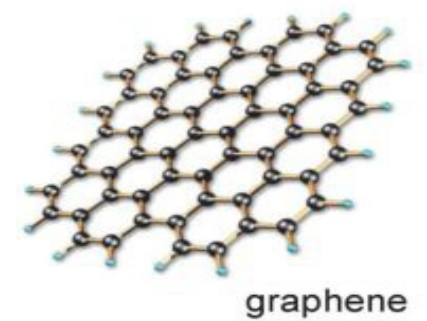

Figura 3. Formas alotrópicas del carbono

\section{Métodos de obtención}

Uno de los actuales problemas a los que se enfrentan los investigadores es la producción de grafeno a escala industrial. Los métodos de obtención de grafeno se ven limitados a entornos solo de laboratorio y se espera que en un corto plazo gracias a los estudios e investigaciones, su producción a escala industrial sea una realidad. Actualmente, la obtención del grafeno se puede dar por cualquiera de los métodos siguientes:

\section{- Exfoliación micromecánica}

El grafeno en estado libre fue obtenido por vez primera en 2004 mediante este método. La exfoliación micromecánica es una técnica simple, y es necesario contar con una superficie limpia de grafito, el cual como mencionamos está constituido por varias láminas superpuestas de grafeno débilmente unidas. Este método consiste en someter el grafito a un raspado fino y luego extraer hojuelas extremadamente delgadas al descascarar repetidamente utilizando cinta adhesiva. La mayor parte de las hojuelas obtenidas por este método son tridimensionales, grafito; sin embargo, entre estas se consiguen también bidimensionales, grafeno. Para la identificación de las láminas de grafeno se utiliza microscopia óptica. Este método de obtención del grafeno es muy rudimentario por el proceso de identificación de láminas viables de grafeno.

\section{- Obtención epitaxial en carburo de silicio}

Consiste en calentar el carburo de silicio ( $\mathrm{SiC}$ ) a temperaturas mayores a 1100 ${ }^{\circ} \mathrm{C}$ para reducir al grafeno. Este proceso produce grafeno epitaxial. Muchas propiedades del grafeno importantes han sido identificadas por este método. Mientras que las propiedades electrónicas de ciertos grafenos epitaxiales de capas múltiples son idénticos a los de una sola capa de grafeno, en otros casos las propiedades se ven afectadas como lo son para las capas de grafeno en grafito. Este efecto es, teóricamente, bien entendido y se relaciona con la simetría de las interacciones entre capas. 


\section{- Reducción del óxido de grafito}

La reducción de óxido de grafito era probablemente históricamente el primer método de síntesis de grafema P. Boehm informó escamas monocapas de óxido de grafeno reducido ya en 1962 . En estos primeros trabajos la existencia de pequeñas escamas monocapa de óxido de grafeno se demostró. La contribución de Boehm fue reconocida, recientemente, por el premio Nobel de la investigación de grafeno, Andre Geim. Sin embargo, la calidad de grafeno producido por reducción de óxido de grafito es menor en comparación por ejemplo con el obtenido por exfoliación mecánica debido a la eliminación incompleta de los diversos grupos funcionales con los métodos de reducción existentes.

\section{- Obtención con metal-carbono de- rretido}

La idea general de este proceso es la disolución de átomos de carbono en el interior de un metal de transición fundido a una cierta temperatura, y luego permitir que el carbono disuelto se precipite a bajas temperaturas como grafeno de una sola capa. El metal se funde, en primer lugar, en contacto con una fuente de carbono. Esta fuente puede ser el crisol de grafito dentro de la cual se lleva a cabo el proceso de fusión o podría ser el polvo de grafito. Se debe mantener la masa fundida en contacto con la fuente de carbono a una temperatura determinada dará lugar a la disolución y la saturación de átomos de carbono en la masa fundida basado en el diagrama de fase binaria de metal-carbono. Al bajar la temperatura, la solubilidad del carbono en el metal fundido disminuye y la cantidad en exceso de carbono se precipitará sobre la parte superior de la masa fundida. La capa flotante puede ser o desnatada, o dejar que se congele para su posterior retiro. Al aplicar este método se obtiene en el sustrato de metal morfologías diferentes incluyendo grafito, grafeno de pocas capas y grafeno. El material compuesto de grafeno-metal podría ser utilizado en materiales de interfaz térmica para aplicaciones de gestión térmica.

\section{- Obtención a partir de nanotubos}

Métodos experimentales para la producción de cintas de grafeno consisten en cortar los nanotubos. Los nanotubos de carbono se cortan por acción del permanganato de potasio y ácido sulfúrico. En otro método, las nanocintas de grafeno se producen mediante grabado en plasma de nanotubos, parcialmente, incrustados en una película de polímero.

\section{- Obtención por grafito por ultraso- nido}

Consiste en la dispersión de grafito en un medio líquido adecuado en el que luego se agitan las partículas con ondas sonoras ultrasónicas. El grafito no exfoliado es finalmente separado del grafeno por centrifugación. Se ha conseguido con este método las concentraciones más altas de grafeno informadas hasta el momento y obtenida por cualquier método.

\section{Propiedades del grafeno}

Es de gran importancia conocer las propiedades del grafeno comparándolas con otras características tanto físicas como químicas de otros materiales, para de esta manera entender que estamos 
ante un material que cambiará la tecnología y la vida del hombre como en un momento determinado lo hizo el silicio y el germanio. Algunas de las cualidades más representativas de este nanomaterial son la conductividad eléctrica, la resistividad y la estabilidad termodinámica. A continuación detallamos las propiedades más estudiadas de este prometedor material.

\section{- Comportamiento metálico y efecto de campo eléctrico}

El carbono es un elemento no metálico que es mal conductor de la electricidad; sin embargo, el grafeno presenta propiedades que corresponden a los metales. Se comporta como semiconductor gap (separación entre la banda de valencia y la banda de conducción) superficial. Esto permite que los conductores de carga puedan ser modulados continuamente entre electrones y huecos en altas concentraciones y con una gran movilidad incluso bajo condiciones ambientales. Esto lo convierte en un excelente conductor.

\section{- Quiralidad}

Debido a la simetría de cristal exhibida por el grafeno sus cuasi-partículas deben ser descritas por funciones de onda de dos componentes en virtud de las contribuciones relativas de las dos subredes en la formación de cada una. Pero el spin en el grafeno indica la subred más que el spin real de los electrones surgiendo lo que es conocido como un pseudoespín. Este pseudoespín nos permite introducir el término de quiralidad, definido como la proyección del pseudoespín en la dirección del movimiento siendo positivo para los electrones y negativo para los huecos.

Esta propiedad es muy importante porque permite explicar muchos procesos electrónicos y dos nuevos efectos cuánticos: una conductividad cuántica mínima en el límite de concentraciones desvanecientes de conductores de carga y una supresión fuerte de efectos de interferencia cuántica.

\section{- Electrones del grafeno. Similitud con fermiones de Dirac sin masa}

Ya que los electrones en el grafeno se comporten como partículas relativísticas sin masa en reposo y viajan a $106 \mathrm{~m} / \mathrm{s}$ requieren ser descritas como partículas relativísticas llamadas fermiones de Dirac carentes de masa. Estas partículas pueden ser visualizadas como electrones que han perdido su masa en reposo o como neutrinos que adquirieron la carga electrónica. El origen de estas partículas se debe a la interacción de los electrones de carbono con el potencial periódico dado por la estructura de panal del grafeno, produciendo cuasipartículas que, a bajas energías, se describen exactamente por la ecuación Dirac, por lo que son llamadas fermiones Dirac carentes de masa, regidas por la siguiente ecuación para la expresión del espectro de energía:

$$
E= \pm \sqrt{2|e| h v^{2} f B}\left(v+\frac{1}{2} \pm \frac{1}{2}\right)
$$

Donde $\mathrm{f}$ es la velocidad del electrón, $\mathrm{v}=0,1,2 \ldots$ es el número cuántico $\mathrm{y}$ el término con $\pm \frac{1}{2}$ está relacionado con la quiralidad. El efecto Hall cuántico 
anómalo es la evidencia más directa para asegurar la existencia de los fermiones de Dirac carentes de masa en el grafeno.

\section{- Efecto Hall cuántico anómalo}

Constituye la mayor prueba de la existencia de los fermiones Dirac carentes de masa en el grafeno. El efecto Hall cuántico es utilizado para determinar tanto el signo como los portadores de carga. Para efectos explicativos conviene que el efecto Hall cuántico se le relacione con el efecto Hall el cual se establece mediante el siguiente procedimiento: al material a investigar por el cual se hace pasar una corriente eléctrica se le aplica perpendi- cularmente un campo magnético provocándose que los portadores de carga se acumulen en un determinado sitio, generándose un campo eléctrico. Midiendo la diferencia de potencial en las dos superficies del material es posible deducir el signo y la densidad de los portadores de carga, presentándose una linealidad entre el campo magnético y la resistencia Hall. El efecto Hall cuántico ofrece, por lo contrario, como característica la no linealidad entre la resistencia de Hall y el campo magnético; es decir, que el efecto Hall cuántico se presenta mediante una serie de escalones al aplicársela en campos magnéticos altos y bajas temperaturas. (Figura 4)

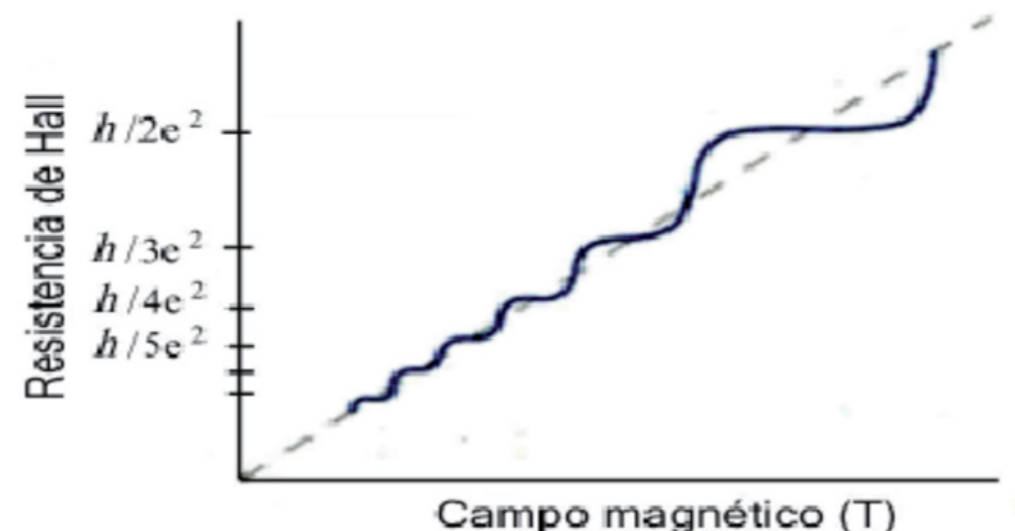

Figura 4. Efecto Hall Cuántico. La línea punteada indica el comportamiento clásico esperado, la continúa el comportamiento cuántico.

\section{- Paradoja de Klein}

De acuerdo con la teoría clásica, una partícula no puede propagarse a través de una región donde su energía potencial es más grande que su energía total. Sin embargo, los electrones obedecen las leyes de la mecánica cuántica, según las cuales el electrón rebotará, efectivamente, contra la barrera, pero además hay una probabilidad finita de que también "atraviese" la barrera en un proceso denominado efec- to túnel y de que aparezca del otro lado. Sorpresivamente, en el caso del grafeno la probabilidad de transmisión del electrón es siempre igual a 1 independientemente de la altura y anchura de la barrera. Esta conducta en electrodinámica cuántica es conocida como la paradoja Klein.

\section{- Efecto piezoeléctrico}

El grafeno podría unirse al selecto grupo de materiales piezoeléctricos que 
generan electricidad cuando se flexionan o se les presiona y viceversa. Abriendo agujeros en una hoja de grafeno dispuestos en puntos específicos de la lámina siguiendo una configuración especial es factible forzar al grafeno a comportarse igual que un material piezoeléctrico.

Las substancias piezoeléctricas son un buen recurso para desarrollos de vanguardia, como por ejemplo la recolección de energía mecánica en fuentes difícilmente aprovechables por otros métodos, o los músculos articules, y también sirven a los ingenieros para fabricar sensores de muy alta precisión.

\section{Aplicaciones del grafeno en el campo energético}

La aplicación del grafeno en el campo energético busca lograr que las fuentes de energía que, actualmente, son utilizadas, sean mejoradas en términos principalmente de eficiencia. Esto se puede conseguir haciendo uso de diversas técnicas y procedimientos que básicamente buscan que los componentes como baterías, celdas solares, supercapacitores y demás dispositivos que forman parte esencial de los dispositivos generadores de energía renovable, logren superar las características técnicas actuales de los dispositivos mencionados. A continuación se detalla cada dispositivo mencionado.

\section{- Baterías}

En esta área, gracias a su conductividad, el grafeno entra para disminuir los tiempos de carga y descarga de baterías. Se ha desarrollado un nuevo ánodo basado en grafeno que puede ser cargado o descargado 10 veces más rápido que los ánodos de grafito que se utilizan actualmente en las baterías de litio. Para crear el material del ánodo, los investigadores tomaron una lámina de óxido de grafeno e introdujeron, intencionalmente, defectos, innumerables grietas, poros y otras imperfecciones. Los iones de litio pueden utilizar las grietas en el óxido de papel de grafeno para atravesar rápidamente la hoja entera, lo que significa cargas y descargas más rápidas de la batería. Resulta muy interesante también la implementación del grafeno a las baterías de níquel-hierro. Inventadas por Edison, constituyen una alternativa barata y segura de las de ácido y plomo, además que tanto el níquel como el hierro son dos materiales muy abundantes.
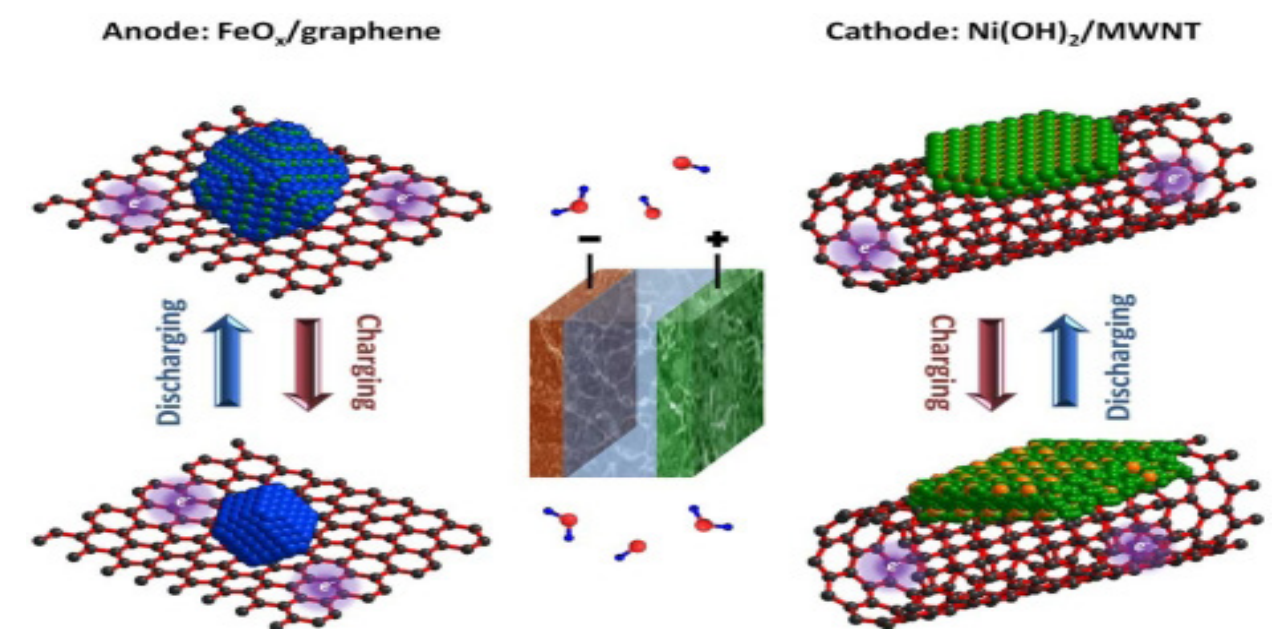

Figura 5. Esquema de la batería Ni-Fe mejorada con capas de grafeno 
Las principales ventajas de las baterías mejoradas residen en su fiabilidad y en su velocidad de carga y descarga. En general, es útil como respaldo de otras baterías o de cualquier aparato eléctrico que pueda requerir de una gran carga en muy poco tiempo. $\mathrm{Su}$ único problema es que pierden en torno a un $20 \%$ de su capacidad tras 800 ciclos de carga y descarga. Esto, que no es tan grave en otro tipo de baterías más lentas, puede ser el principal impedimento para que esta nueva tecnología se asiente.

\section{- Celdas solares}

Se ha alcanzado una eficiencia del 8,6 por ciento con nuevas celdas solares gracias un tratamiento químico en el que se dopa al grafeno con silicio amorfo (TFSA). Dopar con TFSA al grafeno hace que se vuelva mejor conductor y eso incrementa el potencial del campo eléctrico dentro de la célula solar, volviéndola más eficiente en la conversión de la luz del Sol en electricidad. Además, a diferencia de otros materiales para dopar al grafeno que han sido probados en el pasado, la TFSA es más estable; es decir que sus efectos son mucho más duraderos.

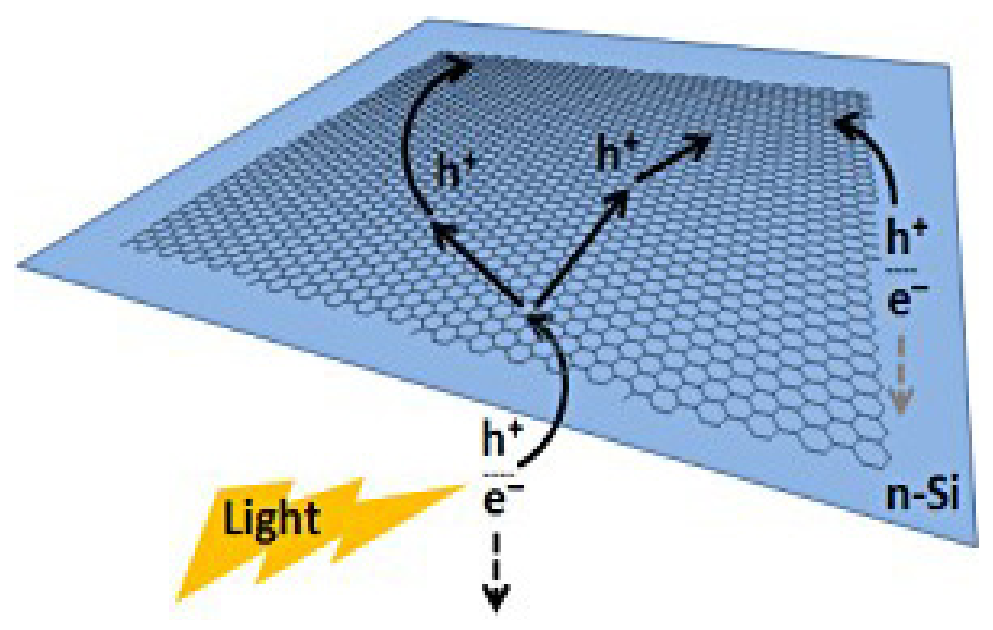

Figura 6. Esquema de celda solar de grafeno dopada con silicio amorfo (TFSA)

El grafeno gracias a que es transparente y flexible tiene una ventaja para ser un componente importante en el tipo de células solares instaladas en los exteriores de edificaciones y en otros medios. Haber mostrado ahora que sus capacidades de generar electricidad a partir de la luz solar pueden ser realzadas por un tratamiento tan simple y barato, ofrece buenas perspectivas para el uso futuro del grafeno en células solares. Se estima que si las células solares con grafeno alcanzaran eficiencias de conversión de un 10 por ciento podrían ser un claro competidor en el mercado de los paneles solares, siempre y cuando sus costes de producción sean mantenidos en un nivel lo bastante bajo. El prototipo de célula solar creado en el laboratorio de la Universidad de Florida fue construido sobre una base rígida de silicio, un soporte que no se considera lo bastante rentable para la producción a gran escala en los términos antedichos de comercialización barata. Pero es factible combinar el uso de grafeno dopado con sustratos más flexibles y más baratos. 


\section{- Supercapacitores}

Un gran avance en el desempeño de los capacitores ha sido alcanzado con el desarrollo de un dispositivo que puede almacenar tanta energía como una batería y es capaz de recargarse en segundos.

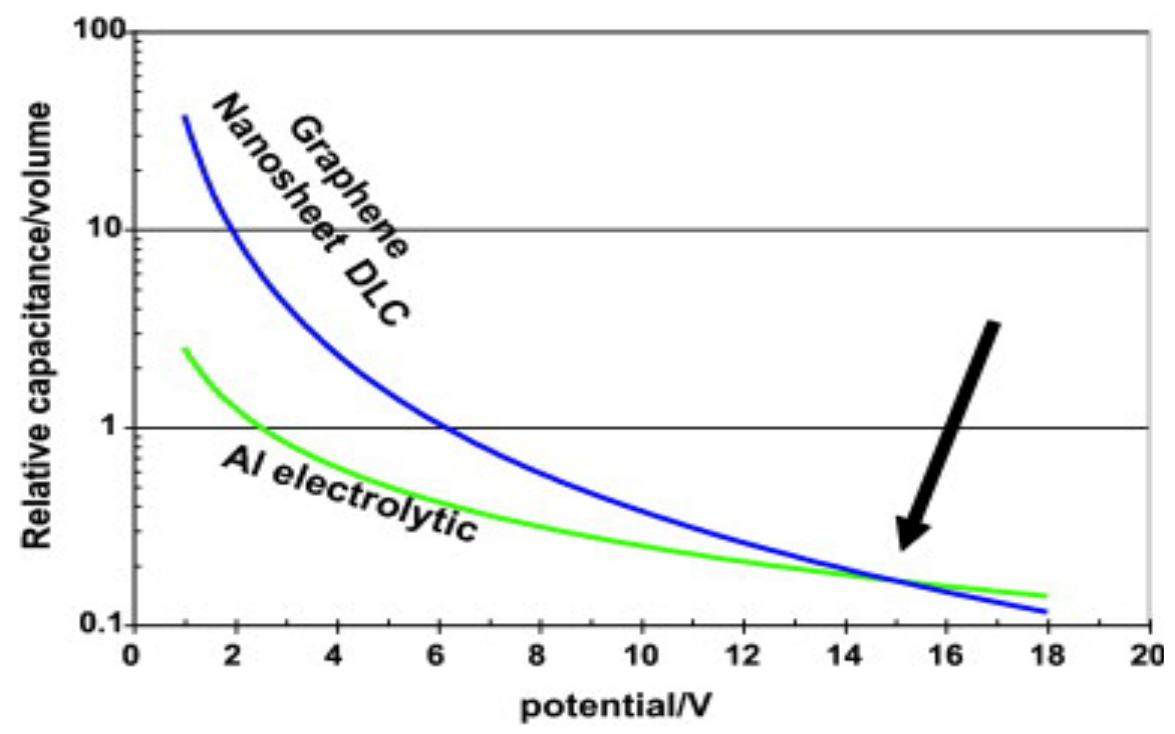

Figura 7. Curva Potencia/V Vs Capacidad Relavita/Vol del Supercapacitor de Grafeno

El supercapacitor basado en grafeno puede almacenar tanta energía por unidad de masa como las baterías de níquel metal hidruro y podrían ser usadas algún día para proporcionar energía a aparatos diversos como teléfonos móviles, cámaras digitales y micro vehículos eléctricos. Con la gran área de superficie de sus electrodos y el espacio extremadamente pequeño que hay entre estos pueden almacenar una gran cantidad de carga eléctrica en un pequeño volumen.

El nuevo dispositivo tiene electrodos hechos de grafeno mezclado con un negro de acetileno llamado Súper $\mathrm{P}$ que actúa como un aditivo conductor y un aglutinante que mantiene todo unido. La mezcla resultante se cubre dentro de la superficie de un colector de corriente y es ensamblado en forma de capacitores del tamaño de una moneda.
La energía que puede ser almacenada por unidad de peso es de $85.6 \mathrm{Wh} /$ $\mathrm{kg}$ a una temperatura regular de 20 a 30 grados Celsius, mientras que a 80 grados Celsius el valor medido fue de $136 \mathrm{Wh} /$ $\mathrm{kg}$, el cual es comparable a las baterías $\mathrm{Ni}$-mh. La mayor ventaja de esta tecnología es que los supercapacitores pueden ser recargados en unos cuantos segundos en lugar de minutos como en el caso de las baterías, lo que constituye un gran avance tecnológicamente hablando.

\section{Discusión}

La información revisada da cuenta de los avances de las investigaciones y estudio de las propiedades del grafeno en el aprovechamiento y mejoramiento de dispositivos que almacenan energía. El grafeno es el material más delgado y ligero hasta la fecha conocido, además a partir 
de este material se están modificando otros materiales para obtener características similares o mejores al grafeno. El siliceno es uno de estos materiales en el cual los estudios iniciales indican que su comportamiento y eficiencia podría superar al grafeno.

La coincidencia entre ambos materiales son los costes de producción a escala industrial, que se espera a un corto plazo superar gracias a las investigaciones y estudio científico de estos materiales. Una nueva era tecnológicamente hablando está muy próxima con este material: la era de los nanomateriales marcará una nueva etapa en la vida del ser humano, como ya lo fue el silicio y el germanio cuando desplazaron a la válvulas de vacío gracias al transistor de silicio, esta vez el grafeno, según las condiciones actuales se dispone a desplazar al silicio.

Los dispositivos experimentales a base de grafeno que almacenan energía vienen obteniendo resultados muy alentadores y todo indica que su utilización en sistemas más complejos de gestión energética está muy próxima.

\section{Conclusiones}

- El grafeno es un nanomaterial bidimensional sintetizado inicialmente en 2004, con propiedades únicas en su clase, que ha despertado el interés de investigadores y de empresarios. Se espera que en un corto plazo gracias al avance en los estudios y pruebas de los investigadores, su producción se masifique a escala industrial.

- Existen múltiples formas para la obtención de grafeno, todas con resultados aceptables y diversos costes de producción. Lamentablemente, los costes presentan el principal inconveniente para la producción de grafeno a escala industrial.

- Las propiedades que presenta el grafeno son excepcionales, lo que hace que este material sea el principal candidato a iniciar una nueva revolución tecnológica teniendo como base la eficiencia energética y el respeto por el medio ambiente. Mientras las pruebas en laboratorio y las investigaciones van avanzando se dan nuevos alcances del aprovechamiento de las propiedades del grafeno.

- La eficiencia energética de los dispositivos que almacenan energía a base grafeno ha sido demostrada en laboratorio por los investigadores, lo que vaticina un futuro promisorio de este material y el desplazamiento de muchos de los componentes, actualmente, utilizados en áreas relacionadas con el quehacer tecnológico.

\section{Referencias}

Bonaccorso, F.,Sun, Z., T. Hasan, A., Ferrari, C. IGraphene Photonics and Optoelec-tronics", Department of Engineering, University of Cambridge, Cambridge, UK
Castro, A. H.; F. Guinea; N. M. R. Peres; K. S. Novoselov \& A. K. Geim (2009). "The Electronic Properties of Graphene", Rev. Mod. Phys. Vol. 81: 109-162. 
Chávez Castillo, M. R. (2012). Propiedades estructurales y termodinámicas del siliceno. (Tesis de Maestría). Instituto de Física "Luis Rivera Terrazas". Benemérita Universidad Autónoma de Puebla.

Geim Andre (2005). Electrons lose their mass in carbon shests, Nature materials (UK) Vol. 438 pp $165-167$

Katsnelson, Mikhail I. (2007).Graphene: carbon in two dimentions, Materials today ( Paises Bajos) Vol. 10, num 1-2, pp.20-27

Novoselov, K. S., A.K. Geim et al. (20 04) Electric field effect in atomica- lly thin carbon film, Science (UK y Rusia) Vol.306, pp. 666-669

Resnick, R., D. Halliday y K.S. Krane(2003) Física CECSA, Mexico $4^{\circ}$ ed. pp. 736.

Rodríguez, C. \& Vasilievna, O. (2007). Propiedades y aplicaciones del grafeno. UANL

Saito, R, G. Dressellhaus y M.S. Dresselhus. (1998) Physical properties of carbon nanotubes, Imperial College press, Singapur, 2a. ed. Pp 24-25 